\title{
La guêpe solitaire Ectemnius nigritarsus (Herrich- Schäffer 1841) retrouvée en Belgique après une éclipse de septante ans (Hymenoptera : Crabronidae)
}

\author{
Jean-Yves Baugnée et Yvan Barbier
}

Service public de Wallonie, Département du Milieu Naturel et Agricole, Avenue de la Faculté, 22, B5030 Gembloux, Belgique. E-mail : jybaugnee@gmail.com

Reçu le 17 Février 2021, accepté le 8 Mars 2021.

La capture d'un mâle d'Ectemnius nigritarsus (Herrich-Schäffer 1841) à Sart-Bernard (commune d'Assesse, province de Namur) le 23 juillet 2019 dans une lande à callune infra-forestière, vient confirmer la présence actuelle de ce Crabronidae sur le territoire belge, où il n'était connu auparavant que par deux anciennes occurrences datées de 1896 et 1946. Les circonstances de l'observation sont décrites et les données sur la biologie et la répartition de cette espèce rare en Europe occidentale sont résumées.

Mots-clés: Ectemnius nigritarsus, guêpe solitaire, Crabronidae, lande à callune, Condroz.

A male of the rare Crabronid wasp Ectemnius nigritarsus (Herrich-Schäffer 1841) was captured on 23 July 2019 in a small forest heathland at Sart-Bernard (commune of Assesse, province of Namur). It confirms the current occurrence of the species in Belgium, where it was previously known only by two former observations in 1896 and 1946. The circumstances of the observation are described and data on the biology and distribution of this rare species are summarized.

Keywords: Ectemnius nigritarsus, solitary wasp, Crabronidae, heathland, Condroz.

\section{INTRODUCTION}

Les landes à bruyères représentent un habitat peu commun et très localisé dans le Condroz namurois où elles ne subsistent plus guère que sous la forme de fragments confinés le plus souvent sur les lisières ou les clairières en forêt et dans certaines carrières. Or, on sait que ce type de végétation, issu la plupart du temps d'anciennes pratiques agro-pastorales, héberge des cortèges d'espèces végétales et animales spécialisées à haute valeur patrimoniale.

Il y a une trentaine d'années, une lande s'est développée à l'est du village de Sart-Bernard (commune d'Assesse, province de Namur) à la faveur de travaux de déboisement d'une enclave forestière appartenant au Bois d'Ausse. A la suite d'une rapide visite en 2014 en compagnie de notre collègue Benjamin Snoeck (DNF) qui nous a révélé l'existence de cette lande, une nouvelle prospection fut programmée le 23 juillet 2019 en vue de préciser les caractéristiques de la végétation actuellement en place et l'intérêt biologique de l'endroit.

C'est à cette occasion que fut constatée la présence de l'Hyménoptère Crabronidae Ectemnius nigritarsus (Herrich-Schäffer 1841), une espèce certes non directement liée aux landes à bruyères, mais qui a ceci de remarquable qu'elle apparaît rare partout en Europe et qu'elle n'a plus été signalée officiellement du territoire belge depuis plus de 70 ans! Les circonstances de cette redécouverte sont décrites dans les lignes qui suivent et les informations relatives à l'espèce sont résumées et commentées. 


\section{LE SITE DE CAPTURE}

Localisée à une dizaine de kilomètres au sud-est de Namur, la lande du Bois d'Ausse $\left(50^{\circ} 24^{\prime} 08.3^{\prime \prime} \mathrm{N}\right.$, $4^{\circ} 58^{\prime} 07.2^{\prime \prime} \mathrm{E}$; altitude $255 \mathrm{~m}$ ) prend place au sein d'une clairière forestière d'environ 70 ares enserrée à l'intersection de trois voies de communication majeures, à savoir la ligne de chemin de fer BruxellesLuxembourg au nord, la Nationale 4 à l'ouest et l'autoroute E411 à l'est (Figure 1). Le site occupe des sols acides de type limono-caillouteux à forte charge gréseuse et à drainage faible, reposant sur un socle de roches du Dévonien inférieur qui caractérise cette partie du plateau condruzien, communément dénommé le Condroz ardennais (ou "Ardenne condruzienne") en raison de similitudes avec l'Ardenne, notamment dans la géologie et le paysage végétal (voir e.a. Schmit et al., 2016). Du point de vue biogéographique, le site se localise en marge nord-ouest de la région continentale d'Europe ${ }^{1}$ et au sein du district phytogéographique mosan.

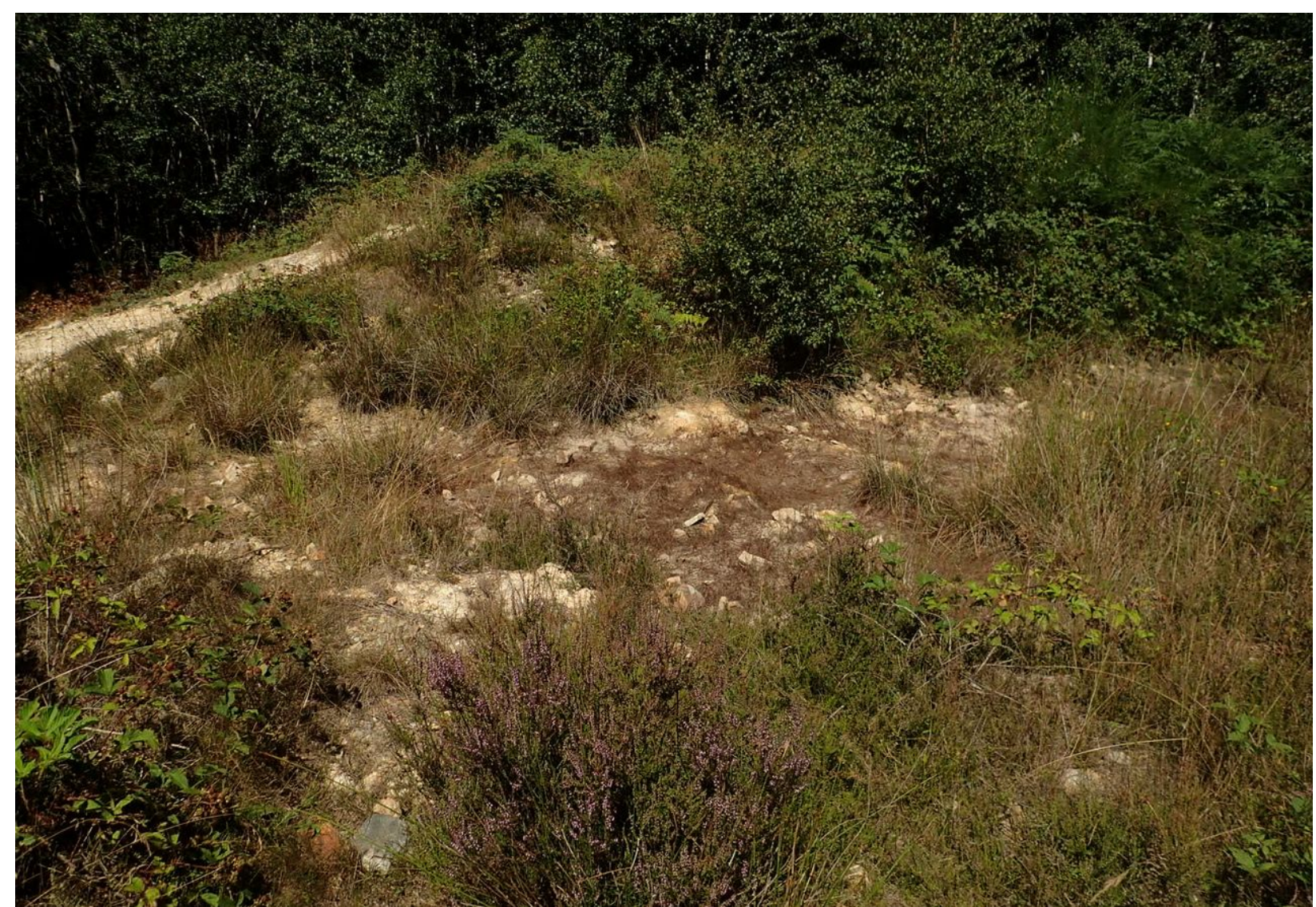

Figure 1: Ectemnius nigritarsus (Herrich-Schäffer 1841), habitat à Sart-Bernard, 23.vii.2019. (C) J.-Y. Baugnée

L'origine de la lande remonte au début des années 1990, lorsque des travaux de déboisement préparatoires à l'implantation d'un complexe cinématographique ont débouché sur une vaste clairière au sein de cette enclave forestière complètement cernée par les voiries. Le projet fut finalement abandonné et la clairière, laissée à son évolution spontanée, s'est vue progressivement recolonisée par divers ligneux, en particulier des bouleaux (Betula pendula Roth et B. pubescens Ehrh). Durant les années 2000, le site intéresse des amateurs de motocross qui, en accord avec le propriétaire de l'époque, y aménagent un circuit temporaire consistant en une succession de bosses, de fosses et de petites mares tout le long d'une piste très sinueuse. Actuellement, les massifs de callune (Calluna vulgaris Hull.) se maintiennent essentiellement en bordure de cette piste, en compagnie d'autres plantes acidophiles comme la fougère aigle (Pteridium aquilinum (L.) Kuhn), la tormentille (Potentilla erecta (L.) Räusch.), le millepertuis

\footnotetext{
${ }^{1}$ Centre thématique européen sur la diversité biologique, Agence européenne pour l'environnement, http://biodiversity.eionet.europa.eu, octobre 2008.
} 
élégant (Hypericum pulchrum L.), la canche flexueuse (Deschampsia flexuosa (L.) Trin.), la laîche à pilules (Carex pilulifera L.), etc. Les ronces (Rubus fruticosus L. s.l.) sont également bien présentes et forment localement des massifs assez denses. Les mares, au nombre d'une vingtaine, sont quant à elles colonisées presque exclusivement par un groupement amphibie à jonc bulbeux (Juncus bulbosus L.).

Le mâle d'Ectemnius nigritarsus capturé le 23 juillet 2019 (Figures 2-4) fut l'unique spécimen contacté ce jour-là, malgré notre présence prolongée sur le site (de 14 à 19h30) et des conditions météorologiques à priori optimales (soleil, vent faible, $25^{\circ} \mathrm{C}$ ). Il survolait des ronces envahissant la lande au niveau de l'ourlet en contact avec la piste de motocross. D'après son comportement, il ne semblait pas particulièrement à la recherche de fleurs à butiner, celles de la ronce étant d'ailleurs largement fanées tandis que les autres floraisons apparaissaient peu abondantes (absence d'ombellifères, e.a.). N'ayant pas été reconnu comme tel sur le terrain, le spécimen fut collecté et son identité spécifique établie au laboratoire et confirmée par Y. Barbier; il figure à présent dans la collection du premier auteur et la donnée originale a été encodée sur le portail d'encodage en ligne du DEMNA-OFFH².

Aucune autre espèce d'Ectemnius n'a été aperçue ce jour-là. En revanche, deux autres Crabronidae intéressants y ont été notés: d'une part Astata boops (Schrank 1781), une espèce bénéficiant d'une protection stricte en Région wallonne $e^{3}$ et qui tend à devenir plus fréquente en ce début du $21^{\text {ème }}$ siècle ; et d'autre part Mimesa bruxellensis Bondroit 1934, dont on recense à peine 30 occurrences belges sur deux siècles, la plupart étant anciennes et seulement trois wallonnes: Hergenrath, 1962, Sampont, 1975 et Hensies de 1988 à 2001 (Banque de Données Fauniques Gembloux-Mons).

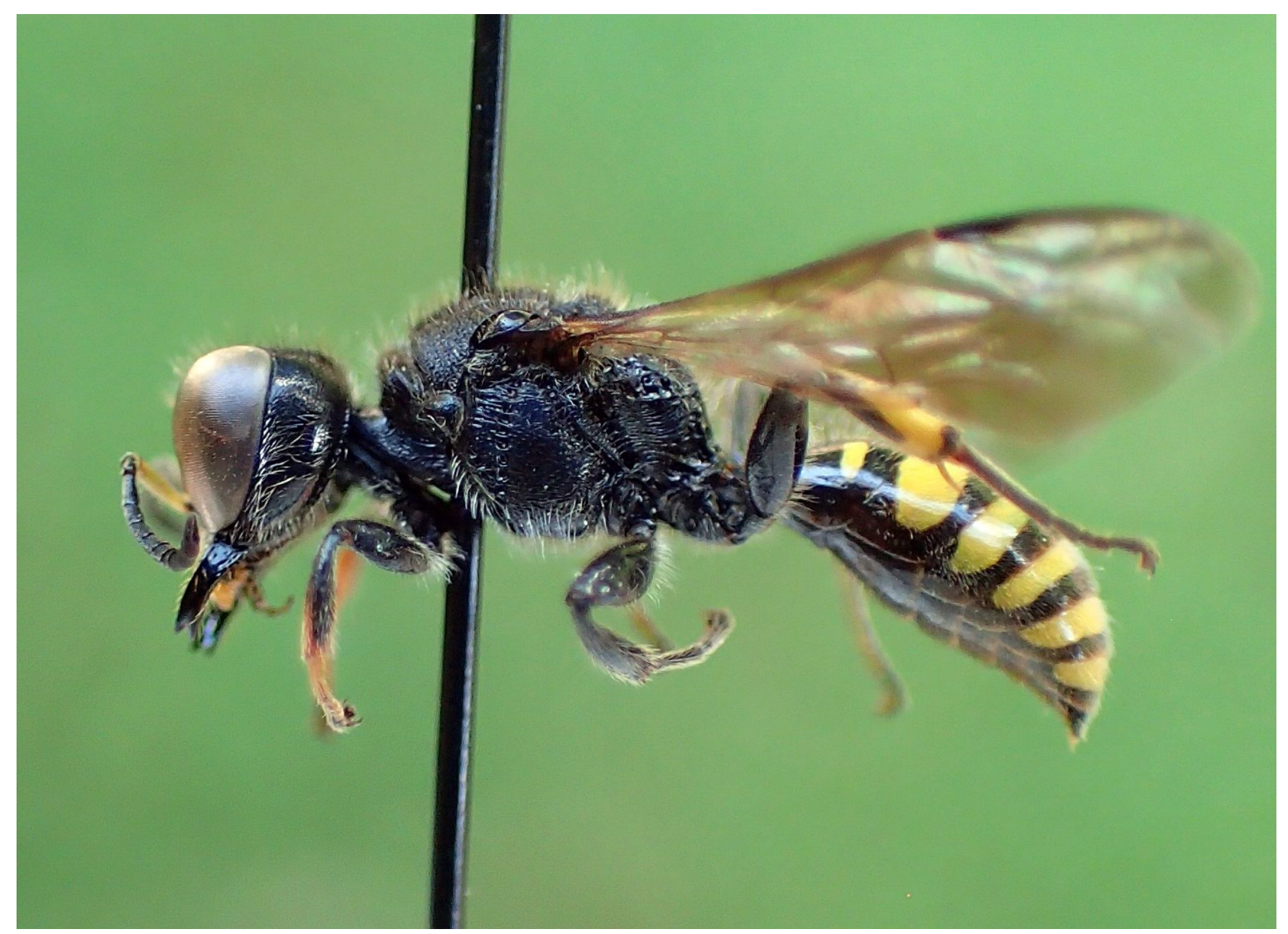

Figure 2: Ectemnius nigritarsus (Herrich-Schäffer 1841), mâle en vue latérale. ㄷ J.-Y. Baugnée

\footnotetext{
${ }^{2} \mathrm{http}: / /$ observatoire.biodiversite.wallonie.be/encodage/

${ }^{3}$ Espèce inscrite à l'Annexe 2b du décret du 6 décembre 2001 modifiant la loi sur la conservation de la nature.
} 


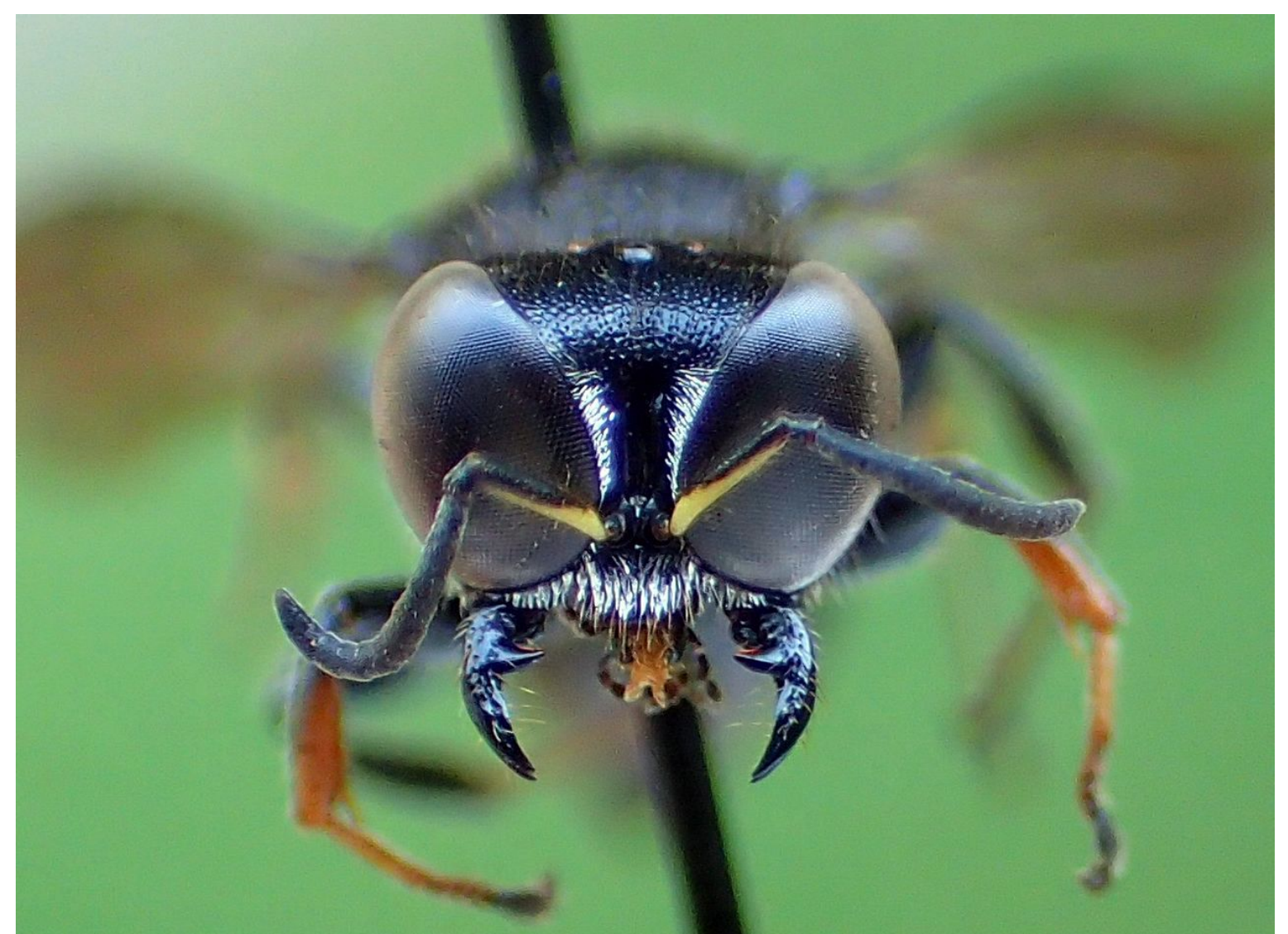

Figure 3: Ectemnius nigritarsus (Herrich-Schäffer 1841), tête du mâle en vue frontale. (C) J.-Y. Baugnée

\section{LE POINT SUR ECTEMNIUS NIGRITARSUS}

Couvrant une grande partie de l'Eurasie, l'aire géographique d'Ectemnius nigritarsus est tellement vaste que quatre sous-espèces ont été décrites pour caractériser des variations chromatiques et morphologiques rencontrées plus particulièrement dans certaines régions d'Asie (Leclercq, 1999), en plus de la sousespèce nominale, seule représentée en Europe. Certains auteurs ont considéré cette espèce comme un élément euro-sibérien mais il serait plus juste de la qualifier de transpaléarctique ou mieux encore d'eurasiatique du fait de sa présence depuis l'Europe jusqu'au Japon en passant par la Russie, l'Inde, la Chine, la péninsule coréenne, les Philippines, l'Australie (Bitsch \& Leclercq, 1993; Blösch, 2000; Kim, 2014; Antropov, 2017). ${ }^{4}$

Par sa silhouette, sa coloration noire bariolée de jaune et une longueur corporelle comprise entre 9 et 12 $\mathrm{mm}$, Ectemnius nigritarsus ressemble superficiellement aux autres espèces du genre Ectemnius Dahlbom 1845, au nombre de 13 en Belgique (Leclercq \& Barbier, 1993). Cependant, c'est la seule dont la mésopleure a un aspect luisant avec une ponctuation simple plus ou moins fine et espacée (au lieu de ponctuée-ridée à réticulée), une caractéristique présente chez les deux sexes et rendant l'espèce en principe aisément reconnaissable.

Les autres critères utiles au diagnostic (et qui caractérisent en même temps le sous-genre Cameronitus Leclercq 1950 dont le seul représentant européen est précisément E. nigritarsus) sont l'absence de fine carène dans le haut du sinus scapal, l'existence d'une très forte dent interne aux mandibules, une pilosité longue et dressée sur le scutum et généralement aussi sur le premier tergite abdominal, l'égale étendue

\footnotetext{
${ }^{4}$ Le Vietnam est régulièrement cité dans la littérature, mais le dernier catalogue en date (Pham et al., 2015) ne fait aucunement mention d'Ectemnius nigritarsus!
} 
des taches jaunes ornant les troisième et quatrième tergites (e.a. Lomholdt, 1984; Leclercq, 1999; Bitsch \& Leclercq, 1993; Bitsch \& Savina, 2017).

Chez les spécimens d'Europe occidentale, le thorax des femelles est entièrement noir mais il peut s'orner de deux petites taches jaunes chez certains mâles. On ajoutera encore que, chez cette espèce, les fémurs des pattes sont entièrement noirs dans les deux sexes et que le $1^{\text {er }}$ tergite typiquement orné d'une bande jaune irrégulière ou de deux à trois petites taches jaunes alignées comme pour le mâle de Sart-Bernard (Figure 4). Enfin, les ocelles sont disposés en triangle équilatéral, ce qui distingue nettement cet Ectemnius par rapport aux Crabro et Crossocerus à dessins jaunes et à mésopleures ponctuées.

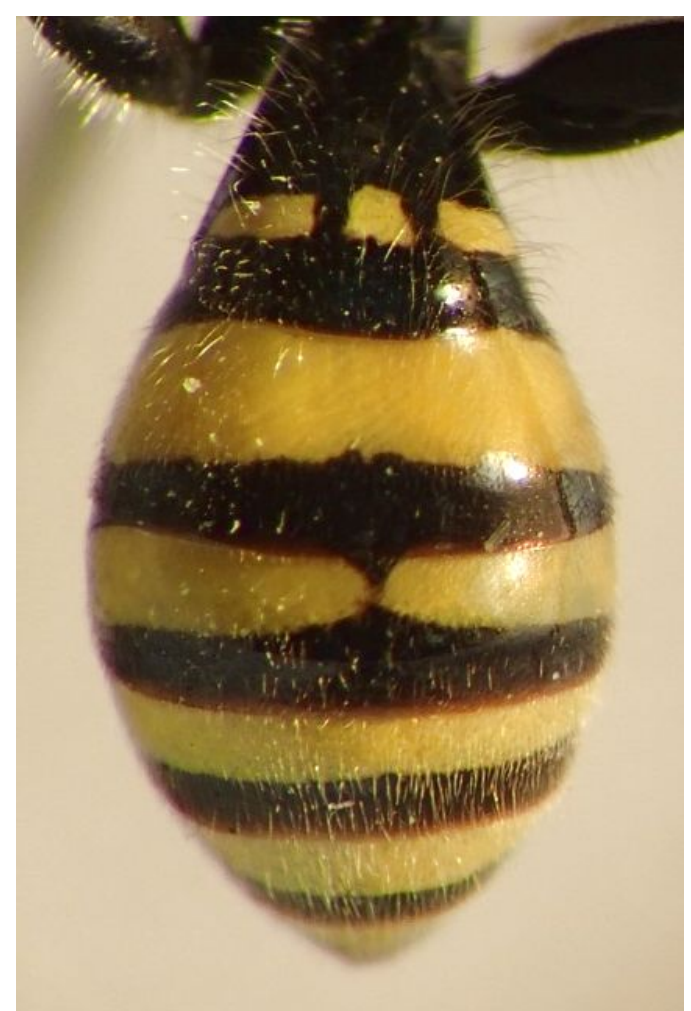

Figure 4 Ectemnius nigritarsus (Herrich-Schäffer 1841), abdomen mâle en vue dorsale. (C) J.-Y. Baugnée`

La pigmentation jaune abdominale est toutefois soumise à une certaine variabilité, aussi bien géographique qu'entre sexes. Celle-ci a été récemment étudiée en France par Bitsch \& Savina (2017) qui notent que, dans l'ensemble, les spécimens français sont davantage marqués de jaune que ceux d'Europe centrale. On remarquera aussi que les mâles sont généralement plus largement tachés de jaune que les femelles et que, sur le $4^{\grave{e}}$ tergite, les taches sont souvent un peu plus étendues que sur le $3^{\grave{e}}$, prenant même souvent l'aspect d'une bande transversale continue.

De plus, chez les mâles, les antennes (qui ne sont composées que de 12 articles comme c'est la règle pour les mâles des Ectemnius!) ne sont pratiquement pas modifiées ni élargies, les $4^{\mathrm{e}}$ et $5^{\mathrm{e}}$ articles étant tout au plus un peu bombés à leur face inférieure et le $6^{\grave{e}}$ article légèrement échancré (Bitsch \& Leclercq, 1993). Toujours chez le sexe mâle, le $7^{\grave{e}}$ tergite est censé porter une petite aire pygidiale arrondie, un caractère parfois peu distinct chez certains spécimens !

Les informations chorologiques relatives à Ectemnius nigritarsus ont été bien résumées par Bitsch \& Leclercq (1993) pour ce qui est du contexte européen en général et du territoire français plus particulièrement. Sur Fauna Europaea (Barbier, 2013), la présence de l'espèce est relevée dans 13 pays: Allemagne, Autriche, Belgique, Espagne, France, Hongrie, Italie, Lettonie, Roumanie, Slovaquie, Suisse, 
Turquie (Thrace orientale) et Ukraine. Il convient d'y ajouter au moins la Moldavie, la Lituanie (Budrys, 2001), la Slovénie (Gogala, 2011), la République Tchèque (Dollfuss, 2004; Bogusch et al., 2011) et la Géorgie $^{5}$ (Dollfuss, 2004). Il existe en outre une mention ancienne mais non confirmée pour la Pologne (Wiśniowski, 2004).

Ceci-dit, pour une espèce aussi répandue et qui occupe des contrées aux climats si contrastés, $E$. nigritarsus manque fort curieusement dans la majeure partie de l'Europe du Nord: c'est le cas aux Iles Britanniques, au Danemark, en Finlande, Suède, Norvège et Islande (Lomholdt, 1984; Hellqvist et al., 2014; Barbier, 2013; Bees, Wasps \& Ants Recording Society, 2020). La guêpe n'y est cependant pas totalement absente puisque des observations sont renseignées pour deux des trois pays baltes. Ainsi, en Lettonie, des femelles isolées sont notées respectivement en 1967 et 1970 dans deux localités distinctes (Tumšs \& Maršakovs, 1970), qui sont du même coup les plus septentrionales connues en Europe. En Lituanie, Budrys (2001) détaille une capture de 1998 dans la forêt de Biržų Giria, à la frontière lettone, laquelle est restée unique à ce jour malgré des recherches régulières menées à travers le pays (E. Budrys, in litt. 16.i.2021).

La distribution d'E. nigritarsus se montre également très lacunaire dans le Bassin méditerranéen, où sa présence n'est attestée qu'en Italie continentale et en Sardaigne (Pagliano \& Negrisolo, 2005), ainsi que dans la Région de Marmara, en Turquie (Y1ldırım et al., 2014). L'occurrence de cette guêpe en Espagne est restée longtemps incertaine, la seule information disponible étant en effet le signalement des Pyrénées par Giner Marí (cité par Bitsch \& Leclercq, 1993). La capture de plusieurs spécimens dans les Asturies, plus exactement à Colunga, en 2009-2011, atteste de l'appartenance définitive de l'espèce à la faune espagnole continentale (Fresno, 2017). De la même région, F. Fresno (in litt. 12.i.2021) nous a confié l'avoir identifié d'une seconde localité située dans le Concejo de Lena, en août 2015.

D'après ce que l'on sait, l'aire européenne d'E. nigritarsus apparaît donc centrée essentiellement sur 1'Europe centrale et orientale, où la plupart des spécialistes s'accordent néanmoins sur son statut d'espèce rare, voire très rare.

Ainsi, en Autriche, cette guêpe est répartie sur diverses régions (Carinthie, Salzbourg, Basse-Autriche, Burgenland, Vienne, Styrie) mais on n'y recense pas plus d'une trentaine de localités (e.a. Zettel et al., 2014).

En Tchéquie, les quelques données répertoriées, soit deux mentions historiques, deux captures en 1981 et une en 2006, sont toutes en Moravie, dans la partie orientale du pays (Bogusch et al., 2011).

En Slovénie, cinq spécimens capturés en 1931-1932 à Podčetrtek constituent les seuls matériaux connus pour ce pays (Gogala, 2011).

En Allemagne, l'espèce est disséminée et globalement rare avec une concentration de stations dans le Bade-Wurtemberg, la Hesse et la Rhénanie-Palatinat, la plupart des données provenant de la vallée du Rhin Supérieur (Schmidt, 1980; Blösch, 2000; Hymenoptera Deutschland, 20206). Elle figure sur la liste rouge nationale dans la catégorie "en danger" (Schmid-Egger, 2010) ainsi que dans les listes rouges les plus récentes de la Rhénanie du Nord-Westphalie comme espèce "extrêmement rare" (Esser et al., 2010), de la Saxe-Anhalt dans la catégorie "disparu ou éteint" (Stolle \& Saure, 2016), et de la Hesse comme espèce "menacée" (Tischendorf et al., 2011). La guêpe n'a été capturée qu'une fois en Saxe-Anhalt, en 1952 (Oehlke, 1970). Plus récemment, elle est renseignée pour la première fois de Basse-Saxe par Theunert (2019) qui a collecté deux femelles en juillet-août 2019 à Einbeck, au sud de Hanovre. Dans la portion ouest du territoire allemand, les données publiées jusqu'ici ne s'écartent guère de la vallée du Rhin: Olpe, en Rhénanie-du-Nord-Westphalie (capture de H. Wolf, 1991, in Woidak, 1996), forêt du Bienwald, près de Karlsruhe (Brechtel, 1986, in Blösch, 2000) et Worms (Reder, 2005) en Rhénanie-

\footnotetext{
${ }^{5}$ Signalé de Gagra, actuellement situé dans la République d'Abkhazie, cf https://fr.wikipedia.org/wiki/Gagra

${ }^{6} \mathrm{http}: / /$ www.aculeata.eu/kartenservice.php?action=arten_d_info_index.php
} 
Palatinat. En outre, un spécimen capturé le 28.vii.1975 à Bad Honnef, au sud de Bonn (Rhénanie-duNord-Westphalie) se trouve dans les collections de Gembloux Agro-Bio Tech (det. J. Leclercq - donnée figurant sur la carte 56 in Leclercq \& Barbier, 1993). Il existe aussi une occurrence plus ancienne à Brodenbach, dans la basse vallée de la Moselle (Aerts, 1955). Mais G. Reder (in litt. 18.i.2021) nous signale l'avoir noté aussi en juin 2015 à Sippersfeld (Rhénanie-Palatinat), à une quarantaine de kilomètres à l'ouest de Worms et donc du Rhin.

En Suisse, De Beaumont (1964) l'indiquait du Plateau en l'estimant peu commun; la carte dynamique générée sur le portail internet du Centre Suisse de Cartographie de la Faune compile seulement 24 données principalement concentrées dans la moitié nord du pays (Plateau suisse et marges du Jura) et parmi lesquelles huit sont postérieures à l'an 2000, la dernière en date provenant du canton de Schaffhouse (obs. A. Müller, 2020) ${ }^{7}$.

Ectemnius nigritarsus apparaît sporadique partout en France et est rarement signalé. La grande majorité des données de la littérature et des collections muséologiques ont été rassemblées par Bitsch \& Leclercq (1993) et cartographiées par Leclercq \& Barbier (1993: carte 56). Cette information montrait sa présence dans 13 départements et donnait une image assez fidèle des connaissances de la répartition de cette espèce à la fin du $20^{\text {ème }}$ siècle. Depuis lors, de nouvelles indications et la confirmation d'anciennes mentions ont été apportées successivement par Bitsch \& Leclercq (2009), Bitsch (2014), et surtout Bitsch \& Savina (2017) qui font le bilan de ce qui est acquis. Au total, l'espèce n'a été notée que dans une petite vingtaine de localités réparties au sein de dix-neuf départements surtout situés dans l'Est, les Alpes du Nord, le Sud-Ouest, mais aussi en Ile-de-France. Dans la Région Grand-Est, elle est citée de Moselle, du Bas-Rhin et de Meurthe-et-Moselle. Plusieurs données du Bas-Rhin proviennent de la vallée du Rhin frontalier possiblement en connexion avec la population du Bade-Wurtemberg. En Bourgogne-Franche-Comté, sa présence est établie au moins dans le Doubs, la Côte-d'Or, la Haute-Saône et le Territoire de Belfort (voir aussi Carminati et al., 2019). Dans le nord de la France, cette guêpe n'aurait été trouvée à ce jour que dans le département de l'Aisne, le 18.vi.2005, à Parfondru, village-clairière situé à $10 \mathrm{~km}$ au sud-est de Laon (Vago, 2005, 2007).

L'espèce n'a encore jamais été rencontrée aux Pays-Bas (Klein \& Lefeber, 2004; W. Klein, in litt. 18.i.2021) ni au Grand-Duché de Luxembourg (N. Schneider, in litt. 13.i.2021).

Quant à la Belgique, les données d'Ectemnius nigritarsus se résument tout au plus à deux captures isolées provenant de la province de Luxembourg (Bitsch \& Leclercq, 1993). C'est Leclercq (1949) qui a dévoilé pour la première fois l'existence de cette espèce sur le territoire, sur la base d'une femelle de la collection de P. de Moffarts étiquetée "Carlsbourg, 9.vii.1896". Deux ans plus tard, le même auteur (Leclercq, 1951) signale une seconde femelle récoltée à Orval, le 2.viii.1946, par R. Vieujant, donnée publiée la même année par l'observateur lui-même (Vieujant, 1951). Curieusement, seule la première occurrence figurait sur la carte 1466 de l'Atlas provisoire des Insectes de Belgique (Leclercq, 1979), la donnée d'Orval apparaissant seulement quinze ans plus tard sur la carte publiée par Leclercq \& Barbier (1993). A notre connaissance, aucun autre enregistrement n'a eu lieu depuis lors, en dehors de l'observation de SartBernard. La répartition des données recueillies en Belgique et dans les régions limitrophes est illustrée sur la carte ci-dessous (Figure 5).

\footnotetext{
${ }^{7} \mathrm{https}$ //lepus.unine.ch/carto-old/index.php?nuesp=58775\&rivieres $=$ on \&lacs $=$ on \&hillsh $=$ on $\& y e a r=1990$
} 


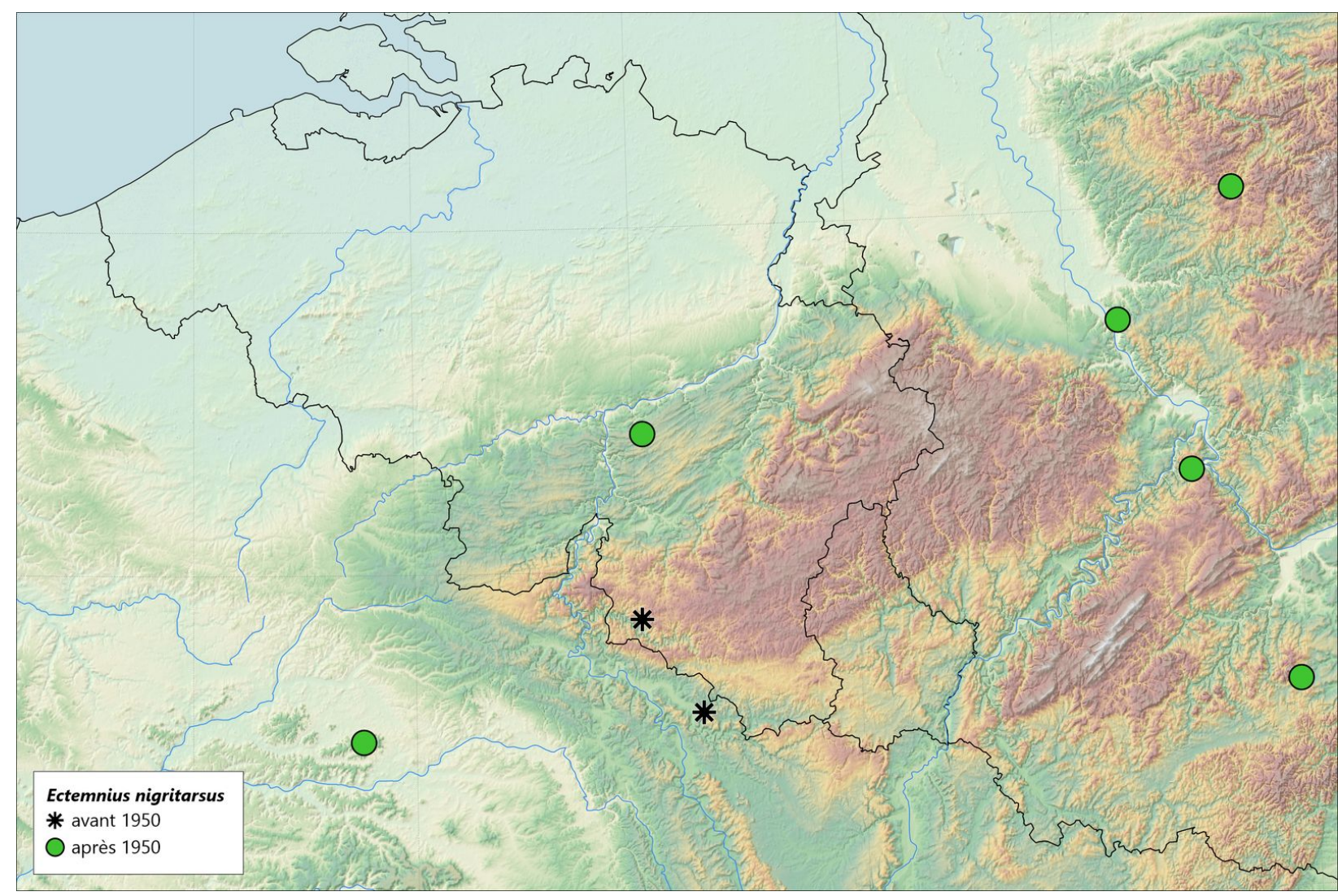

Figure 5: Distribution des observations d'Ectemnius nigritarsus (Herrich-Schäffer 1841) en Belgique

Les exigences écologiques d'Ectemnius nigritarsus sont encore insuffisamment documentées mais la plupart des données de la littérature indiquent qu'il s'agit d'une espèce avant tout forestière liée aux massifs feuillus et à leurs abords directs, aussi bien en climat océanique que dans des secteurs très continentaux.

En Europe de l'Ouest et notamment en France, sa présence a été relevée principalement dans des régions boisées plus ou moins humides, souvent au voisinage de cours d'eau, aux étages collinéen et montagnard, depuis le niveau de la mer jusqu'à $1000 \mathrm{~m}$, rarement au-delà (2000 m max.). Cependant, les précisions quant au biotope occupé font souvent défaut.

Dans les Pyrénées, en Ariège, l'une des observations décrites par Bitsch \& Savina (2017) a été réalisée à $700 \mathrm{~m}$ d'altitude au bord de la retenue d'eau du village de Bonac qui s'inscrit dans une vallée montagneuse aux flancs couverts de forêts. Les autres captures faites à Sinsat, à quelques kilomètres de là, en bordure d'une prairie de fauche, ont pour cadre un paysage bocager à semi-forestier au fond de la vallée de l'Ariège, à $515 \mathrm{~m}$ d'altitude.

Dans le Bas-Rhin, les données proviennent surtout des forêts de la vallée du Rhin limitrophe avec le Bade-Wurtemberg. Au nord de la ville de Strasbourg, les collectes successives effectuées par M. Klein sur une période s'étalant de 1938 à 1971, ont sans doute eu lieu dans la forêt alluviale de la Robertsau (lieu déduit d'après l'indication de Bitsch \& Savina, 2017). A une vingtaine de kilomètres plus en aval, l'espèce est également détectée dans la réserve naturelle de la Forêt d'Offendorf, un vestige de forêt alluviale parcouru d'anciens bras du Rhin (Conservatoire des Sites Alsaciens, $2011^{8}$ ).

En amont de Strasbourg, sur l'ile de Rhinau, Treiber (2010) a encore trouvé une femelle dans une clairière d'un bois rivulaire composé de saules blancs et de résineux. En Haute-Saône, sur la commune de Breurey-

${ }^{8}$ Observation de R. Treiber en 2006, non reprise dans la liste de Bitsch \& Savina (2017) 
les-Faverney, une étude d'impact réalisée durant les années quatre-vingt (Javey, 1990) ${ }^{9}$ a révélé que $E$. nigritarsus figurait parmi les Aculéates remarquables qui sont apparus seulement deux ou trois ans après l'aménagement écologique d'anciennes gravières avec, en corollaire, l'apparition d'une mosaïque de milieux aquatiques, amphibies et terrestres (voir aussi Lemoine, 2015).

La femelle recueillie par Vago (2005) près de Laon, dans les Hauts-de-France, volait parmi la végétation herbacée au sein d'un petit bois feuillus, probablement en quête de proies.

En Allemagne et dans d'autres régions d'Europe centrale, l'espèce semble affectionner tout spécialement les boisements alluviaux thermophiles, notamment le long du Rhin supérieur, mais elle serait plus généralement liée aux forêts feuillues riches en bois mort, que ce soit en plaine ou en montagne (e.a. Blösch, 2000; Tischendorf et al., 2011).

Dans le nord de l'Espagne, le milieu occupé dans les deux localités asturiennes récemment découvertes est composé de prairies ponctuées de chênes, de châtaigniers et de frênes ainsi que d'arbustes divers, celle de Colunga étant située en bord de mer et donc sous forte influence atlantique, alors que Lena se trouve plus à l'intérieur de la cordillère Cantabrique (F. Fresno, in litt. 12.i.2021).

Certaines localités correspondent parfois à des biotopes isolés au milieu de vastes espaces cultivés ou urbanisés. Un exemple intéressant est celui de la "Bosco della Fontana", une réserve naturelle de la plaine du Po, dans le nord de l'Italie. Cette forêt humide de 230 ha, dominée par le chêne pédonculé, est complètement perdue au milieu des cultures intensives et des industries, très loin de tout autre massif boisé (mais bordé par quelques plans d'eau). Ce qui n'empêche pas E. nigritarsus d'y évoluer, comme l'a montré une campagne de piégeage par tente Malaise en 2008 visant à comparer les communautés de Sphéciformes de la canopée et du sous-bois: cinq spécimens y furent collectés dans trois pièges sur quatorze, tous situés dans le sous-bois (Di Giovanni et al., 2017). On peut s'interroger ici sur le caractère relictuel de cette population qui a pu se perpétuer grâce au caractère humide du site et à une gestion favorable au maintien de bois mort.

Cet Ectemnius peu commun n'a pratiquement jamais été l'objet d'études éthologiques si bien que de larges pans de sa biologie demeurent obscurs. Les quelques observations dont on dispose aujourd'hui ont été résumées par Tsuneki (1960) pour la sous-espèce japonaise E. n. munakatai (Tsuneki 1947), et par Lomholdt (1984), Bitsch \& Leclercq (1993), Blösch (2000) et plus récemment Bitsch \& Savina (2017) pour la sous-espèce nominale. Les femelles de la plupart des Ectemnius de nos régions nidifient au sein de galeries creusées par d'autres insectes dans le bois mort ou, plus rarement, dans les tiges à moelle (notamment de ronces et de sureaux), et elles approvisionnent les cellules larvaires avec des Diptères adultes. Hélas, ce qu'on sait au sujet d'Ectemnius nigritarsus est extrêmement limité et repose sur des observations sommairement décrites dans la littérature. Néanmoins, la plupart tendent à montrer que la femelle affectionnerait tout particulièrement les pièces de bois jonchant le sol et dont l'état de décomposition est plus ou moins avancé (Bitsch \& Savina, 2017). En fait, Tsuneki (1960) semble être le seul auteur à avoir pu examiner l'intérieur d'un nid et identifier les proies emmagasinées par la femelle, mais il concerne la sous-espèce japonaise: ces proies étaient des Diptères Brachycères Anthomyiidae, Syrphidae, Tachinidae, Therevidae et Muscidae. En outre, un fait étonnant, mais peut-être simplement accidentel et à confirmer, est l'observation d'une femelle transportant un papillon nocturne au mois de juillet 2004 en Ligurie, au nord-ouest de l'Italie (Pagliano, 2009).

D'autre part, on ne s'étonnera pas pour une espèce aussi peu investiguée, de la quasi absence de données relatives aux parasitoïdes. Parmi les cleptoparasites les mieux connus, il est vrai que les Chrysididae, ou guêpes-coucous, sont rarement renseignés chez les Ectemnius en général (Wiesbauer et al., 2020). Des Hyménoptères Ichneumonidae sont souvent obtenus par élevage de nids de Crabronidae (voir e.a. Yu et al., 2005) mais seule une espèce, le Pimplinae Perithous albicinctus (Gravenhorst, 1829), a été citée

\footnotetext{
${ }^{9}$ Cette indication se rapporte sans doute au mâle de Mersuay pris le 19.viii.1982 par J.-Y. Cretin (cité par Bitsch \& Savina, 2017).
} 
comme ectoparasite des larves d'E. nigritarsus (Constantineanu \& Constantineanu, 1968). Certains Diptères sont aussi associés aux Crabronidae, dont des Anthomyiidae du genre Eustalomyia Kowarz, 1873 et des Sarcophagidae de la sous-famille Miltogramminae, mais aucune espèce n'est signalée spécifiquement pour E. nigritarsus (Gauss, 1997).

Comme beaucoup d'autres Crabronidae, cette guêpe a une phénologie estivale s'étalant de mai à septembre, voire octobre. Les individus des deux sexes visitent les fleurs à la recherche du nectar nécessaire à leur métabolisme, sur lesquelles les femelles trouvent aussi sans doute une partie des proies destinées à l'alimentation de leurs larves. Les mentions de visites florales restent toutefois peu nombreuses et se rapportent majoritairement à des Apiacées, à savoir l'angélique sauvage (Angelica sylvestris L.), la berce commune (Heracleum sphondylium L.) et le panais (Pastinaca sativa L.). Mais des individus isolés ont aussi été notés sur inflorescences du sureau yèble (Sambucus ebulus L.) et de la renouée du Japon (Reynoutria japonica Houtt.) [= Fallopia japonica (Houtt.)] (Bitsch \& Leclercq, 1993; Blösch, 2000; Bitsch \& Savina, 2017). Nous pouvons y ajouter deux données inédites: une femelle visitant un solidage (Solidago sp.) en juin 2015 à Sippersfeld, en Rhénanie-Palatinat (G. Reder, in litt., 18.i.2021), et un mâle collecté sur la renouée bistorte (Bistorta officinalis Delarbre) [= Persicaria bistorta (L.)] à Eyne, dans les Pyrénées Orientales (La Font del Sastre, alt. 1613 m, 06.vii.2017, leg. et coll. X. Van Achter, det. Y. Barbier). Notons que cette dernière localité est seulement la quatrième signalée pour les Pyrénées françaises et constitue une première pour le département, complétant ainsi la liste dressée par Bitsch \& Savina (2017).

Précisons encore que la littérature renferme diverses données d'interception par pièges automatiques (bacs jaunes et tentes Malaise), mais les effectifs collectés par ces méthodes sont toujours peu élevés - le plus souvent 1-5 ex. - comme pour les captures au filet. Celles-ci n'apportent aucun élément probant sur les capacités de déplacement de cette guêpe. Dans l'étude de Di Giovanni et al. (2017), les quelques spécimens analysés ont tous été piégés dans le sous-bois et aucun dans la canopée.

\section{DISCUSSION}

Malgré son ample répartition géographique et en dépit d'une forte augmentation générale de la pression d'observation, Ectemnius nigritarsus demeure une espèce rarement rencontrée. De fait, si sa présence est attestée dans pas moins de dix-sept pays européens, le nombre total d'occurrences répertoriées à ce jour sur ce continent ne dépasserait guère les 150 ! A l'échelle de la Belgique aussi, l'espèce est une véritable rareté avec seulement deux mentions historiques connues et plus aucun signalement au cours des 70 dernières années. Dans ce contexte, la découverte de cette guêpe solitaire en 2019 dans une lande forestière du Condroz namurois est donc aussi inattendue que remarquable, d'autant que les signalements les plus proches proviennent du département français de l'Aisne, à plus de $150 \mathrm{~km}$ de Sart-Bernard, et du land allemand de Rhénanie-Palatinat, au minimum à $220 \mathrm{~km}$ de là (Figure 5).

Il est toujours délicat d'interpréter une observation ponctuelle et d'attribuer un quelconque statut à une espèce si peu étudiée et aussi mal cernée du point de vue éthologique. La taille proportionnellement grande et la coloration assez vive des adultes d'Ectemnius nigritarsus devraient plutôt leur donner une certaine visibilité sur le terrain, contrairement à d'autres Crabronidae tout petits et noirs. En revanche, plusieurs facteurs, dont certains doivent certainement nous échapper, sont susceptibles d'alimenter ou d'accentuer cette impression de rareté. Evoquons notamment des exigences écologiques étroites qui confinerait l'insecte à des habitats spécifiques peu prospectés (forêts alluviales en particulier). Tischendorf et al. (2011) ont d'ailleurs avancé l'hypothèse selon laquelle la guêpe passerait inaperçue parce qu'elle évoluerait essentiellement au cœur des forêts, là où, selon ces auteurs, les hyménoptéristes vont rarement prospecter. On pourrait aussi avancer une autre explication relative à la démographie de l'espèce, supposée peu féconde et produisant des populations très clairsemées et localisées, mais aucune donnée précise n'a été trouvée à ce sujet. La détection des individus pourrait aussi être biaisée par une période d'activité journalière limitée ou inhabituelle, mais ici aussi les informations manquent. En outre, d'après le peu que l'on sait, les femelles nicheraient préférentiellement dans du bois pourri au sol, plus ou moins dissimulé sous la végétation (Bitsch \& Savina, 2017). Ce substrat, inhabituel pour un Ectemnius, 
serait ainsi plus facilement négligé par les observateurs. De plus, s'il venait à être confirmé, ce trait comportemental limiterait la possibilité de rencontrer l'espèce autour des nichoirs à insectes, installations très en vogue depuis quelques années et qui ont déjà permis la découverte d'Hyménoptères rares auparavant très discrets car dilués dans la nature. L'observation de Sart-Bernard n'apporte hélas aucune information utile du point de vue éthologique. A cet égard, il serait instructif d'approfondir les recherches afin de préciser l'importance de la population locale et la présence éventuelle de l'espèce dans les massifs forestiers environnants.

Quoiqu'il en soit, l'existence d'Ectemnius nigritarsus vient souligner le grand intérêt biologique et patrimonial de la lande du Bois d'Ausse. Nous plaidons d'ailleurs pour la mise en place rapide de mesures destinées à assurer le maintien de cette lande, via par exemple un statut de réserve naturelle, tout en veillant à développer sur le site et dans la forêt environnante le stock de bois mort à différents degrés de décomposition, sur pied et au sol, afin de favoriser la faune saproxylique à laquelle appartient $E$. nigritarsus. Plus généralement, ces mesures devraient s'appliquer d'urgence aux autres fragments de landes qui subsistent dans la région et qui sont, pour la plupart, situés hors zones protégées.

\section{REMERCIEMENTS}

Notre reconnaissance s'adresse à Benjamin Snoeck (DNF) qui nous a signalé l'existence de la lande du Bois d'Ausse tout en attirant notre attention sur son intérêt biologique potentiel. Nous remercions également Michel Fautsch pour les informations transmises sur ce site, ainsi que Christian Schmid-Egger, Eduardas Budrys, Fernando Fresno, Gerd Reder, Wim Klein, Reiner Theunert et Nico Schneider pour leurs échanges utiles, et enfin Xavier Van Achter pour le prêt de spécimens.

\section{BIBLIOGRAPHIE}

Aerts W., 1955. Grabwespen (Sphegidae) und andere Hymenopteren des Rheinlandes. Decheniana, 108, 55-68.

Antropov A.V., 2017. Family Crabronidae. Subfamily Crabroninae, p. 225-245. In: Belokobylskij S.A. \& Lelej A.S. (eds). Annotated Catalogue of the Hymenoptera of Russia, Vol. I. Symphyta and Apocrita: Aculeata. Proceedings of the Zoological Institute of the Russian Academy of Sciences Supplement, 6, 1-475.

Barbier Y., 2013. Fauna Europaea: Crabronidae. In: Mitroiu, M.-D., Fauna Europaea: Hymenoptera, Apoidea. Fauna Europaea version 2017.06, https://fauna-eu.org

Bees, Wasps \& Ants Recording Society, 2020. Ectemnius. https://www.bwars.com/category/taxonomichierarchy/ectemnius-crabroninae-crabronidae-wasp

Bitsch J., 2014. Sphéciformes nouveaux ou peu connus de la faune de France et d'Europe occidentale (Hymenoptera, Aculeata). Bulletin de la Société entomologique de France, 119 (3), 391-419.

Bitsch J. \& Leclercq J., 1993. Hyménoptères Sphecidae d'Europe occidentale. Vol. I. Faune de France, 79, 325 p.

Bitsch J. \& Leclercq J., 2009. Compléments au volume 1 des Hyménoptères Sphecidae d'Europe occidentale (Faune de France 79). Bulletin de la Société entomologique de France, 114 (2), 211-244.

Bitsch J. \& Savina H., 2017. Ectemnius nigritarsus (Herrich-Schäffer), espèce sporadique de la faune eurasienne (Hymenoptera, Crabronidae). Bulletin de la Société entomologique de France, 122 (2), 143-150.

Blösch M., 2000. Die Grabwespen Deutschlands. Sphecidae s.str., Crabronidae. Lebensweise, Verhalten, Verbreitung. Die Tierwelt Deutschlands, 71. Teil, Goecke \& Evers, Keltern, 480 p.

Bogusch P., Straka J., Karas Z., Macek, J., Dvořák, L., Vepřek, D. \& Řiha, M., 2011. Faunistic records from the Czech Republic - 310. Hymenoptera : Apocrita. Klapalekiana, 47, 91-99.

Budrys E., 2001. Five new for Lithuanian fauna species of Sphecidae and Crabronidae (Hymenoptera, Apoidea). Acta Zoologica Lituanica, 11 (4), 388-390.

Carminati J., Mora F. \& Cretin J.-Y., 2019. Liste commentée des Hyménoptères "Sphéciformes" de Franche-Comté (Ampulicidae, Crabronidae, Sphecidae). Conservatoire Botanique National de Franche-Comté - Observatoire régional des Invertébrés, $33 \mathrm{p}$. 
Conservatoire des Sites Alsaciens, 2011. Plan de gestion de la Réserve Naturelle Nationale de la Forêt d'Offendorf 2012-2016, $257 \mathrm{p}$.

Constantineanu M. I. \& Constantineanu R. M., 1968. Contributions à l'étude du genre Perithous (Hym., Ichneum.) de la R. S. Romania. Zoologischer Anzeiger, 180 (3/4), 228-258.

De Beaumont J., 1964. Hymenoptera: Sphecidae. Insecta Helvetica, Fauna 3, 169 p.

Di Giovanni F., Mei M. \& Cerretti P., 2017. Vertical stratification of selected Hymenoptera in a remnant forest of the Po Plain (Italy, Lombardy) (Hymenoptera: Ampulicidae, Crabronidae, Sphecidae). Fragmenta entomologica, 49 (1), 71-77.

Dollfuss H., 2004. The Crabroninae Wasps of "Biologiezentrum Linz" - Collection in Linz, Austria (Hymenoptera, Apoidea, Crabronidae), Part 1. Linzer biologische Beiträge, 36 (2), 761-784.

Esser J., Fuhrmann M. \& Venne C., 2010. Rote Liste und Gesamtartenliste der Wildbienen und Wespen (Hymenoptera: Apidae, Crabronidae, Sphecidae, Ampulicidae, Pompilidae, Vespidae, Tiphiidae, Sapygidae, Mutillidae, Chrysididae) Nordrhein-Westfalens. Ampulex, 2, 5-60.

Fresno F., 2017. Aportación al conocimiento de la distribución de especies interesantes de Apoidea Spheciformes en España (Hymenoptera: Ampulicidae, Sphecidae y Crabronidae). Revista gaditana de Entomología, 8 (1), $145-$ 161.

Gauss R., 1997. Eiablage parasitoider Dipteren an Aculeaten. Bembix, 9, 25-28.

Gogala A., 2011. Sphecid wasps of Slovenia (Hymenoptera: Ampulicidae, Sphecidae and Crabronidae). Scopolia, 73, 1-39.

Hellqvist S., Abenius J. \& Norén L., 2014. Provinsförteckning för de svenska arterna i familjerna Ampulicidae, Sphecidae och Crabronidae (Hymenoptera). Entomologisk Tidskrift, 125 (1-2), 77-94.

Javey C., 1990. Mairie de Breurey-les-Faverney - Centre de loisirs et d'initiation à la nature du domaine des Martins-Pêcheurs. Projet d'extraction de granulats alluvionnaires nécessaire à la création de plans d'eau. Etude d'impact sur l'environnement. Bureau de Recherches Géologiques et Minières, 74 p. + annexes.

Kim J.-K., 2014. Annotated catalog of the series Spheciformes (Hymenoptera: Apoidea) from the Korean Peninsula. Journal of Asia-Pacific Biodiversity, 7, 415-456.

Klein W.F. \& Lefeber V., 2004. Crabronidae - graafwespen (p. 356-430). In: Peeters T.M.J., van Achterberg C., Heitmans W.R.B., Klein W.F., Lefeber V., van Loon A.J., Mabelis A.A., Nieuwenhuijsen H., Reemer M., de Rond J., Smit J. \& Velthuis H.H.W. De wespen en mieren van Nederland (Hymenoptera: Aculeata). Nederlandse Fauna 6. Nationaal Natuurhistorisch Museum Naturalis, Leiden, KNNV Uitgeverij, Utrecht \& European Invertebrate Survey - Nederland, Leiden, $507 \mathrm{p}$.

Leclercq J., 1949. Notes détachées sur les Hyménoptères Aculéates de Belgique (4-8). Bulletin et Annales de la Société Entomologique de Belgique, 85, 180-183.

Leclercq J., 1950. Notes systématiques sur les Crabroniens pédonculés (Hymenoptera Sphecidae). Bulletin de l'Institut royal des Sciences naturelles de Belgique, 26 (15), 1-19.

Leclercq J., 1951. Catalogue synonymique des Crabroninae (Hym. Sphecidae) de la Belgique. Bulletin de l'Institut royal des Sciences naturelles de Belgique, 27 (28), 1-20.

Leclercq J., 1979. Atlas provisoire des Insectes de Belgique. Hymenoptera Sphecidae (partim). Cartes 1401 à 1481. Cartographie des Invertébrés Européens, Faculté des Sciences agronomiques de l'Etat, Gembloux.

Leclercq J., 1999. Hyménoptères Sphécides Crabroniens du genre Ectemnius Dahlbom, 1845. Espèces d'Asie et d'Océanie et groupes d'espèces de la faune mondiale. Notes fauniques de Gembloux, 36, 3-83.

Leclercq J. \& Barbier Y., 1993. Atlas de répartition des Crabroniens de France et des régions limitrophes (Hymenoptera, Sphecidae, Crabronini). Notes fauniques de Gembloux, 27, 1-94.

Lemoine G., 2015. Les carrières de sable : une opportunité pour les abeilles solitaires. Établissement Public Foncier Nord - Pas de Calais \& UNPG, Paris, 140 p.

Lomholdt O., 1984. The Sphecidae (Hymenoptera) of Fennoscandia and Denmark. Fauna Entomologica Scandinavica, Volume 4, 2nd edition, 452 p.

Oehlke J., 1970. Beiträge zur Insekten-Fauna der DDR: Hymenoptera - Sphecidae. Beiträge zur Entomologie, 20 $(7 / 8), 615-812$. 
Pagliano G., 2009. Segnalazioni inedite di Sphecidae (Hymenoptera) per il Piemonte e altre regioni italiane. Revista Piemontese di Storia Naturale, 30, 173-192.

Pagliano G. \& Negrisolo E., 2005. Hymenoptera Sphecidae. Fauna d'Italia, 40, i-xi + 1-559.

Pham P. H., Kumar P. G. \& Danilov Y. N., 2015. Distributional checklist of sphecid wasps (Hymenoptera: Ampulicidae, Sphecidae, Crabronidae) from Vietnam. Linzer biologische Beiträge, 47 (2), 1581-1599.

Reder G., 2005. Ergänzungen zur Hymenopterenfauna von Rheinland-Pfalz: Erste Nachweise von Miscophus eatoni S., Mimumesa beaumonti (V. Lith) (Sphecidae) und Chrysis sexdentata Chr. (Chrysididae) (Hymenoptera: Aculeata et Chalcidoidea). Fauna Flora Rheinland-Pfalz, 10 (3), 927-969.

Schmid-Egger C., 2010. Rote Liste der Wespen Deutschlands Hymenoptera Aculeata: Grabwespen (Ampulicidae, Crabronidae, Sphecidae), Wegwespen (Pompilidae), Goldwespen (Chrysididae), Faltenwespen (Vespidae), Spinnenameisen (Mutillidae), Dolchwespen (Scoliidae), Rollwespen (Tiphiidae) und Keulhornwespen (Sapygidae). Ampulex, 1, 5-39.

Schmidt, K., 1980. Materialien zur Aufstellung einer Roten Liste der Sphecidae (Grabwespen) BadenWürttembergs. II. Crabronini. Veröffentlichungen für Naturschutz und Landschaftspflege in BadenWürttemberg, 50/52, 309-398.

Schmit F., Rekk S. \& Hallet V., 2016. Patrimoine géologique et pédologique en province de Namur. 500 millions d'années de façonnement de notre paysage. Fondation Gouverneur René Close, 39 p.

Stolle E. \& Saure C., 2016. Wespen (Hymenoptera: Aculeata). Bestandssituation. In: Frank D. \& Schnitter P. (Hrsg.), Pflanzen und Tiere in Sachsen-Anhalt. Ein Kompendium der Biodiversität. Natur + Text, Rangsdorf, p. 910-929.

Theunert R., 2019. Zur Fauna der Hautflügler (Hymenoptera) des Märchenwaldes im Stadtforst Einbeck. Bericht Umwelt \& Planung Dr. Theunert, Hohenhameln, 17 p.

Tischendorf S., Frommer U. \& Flügel H.-J., 2011. Kommentierte Rote Liste der Grabwespen Hessens (Hymenoptera: Crabronidae, Ampulicidae, Sphecidae) - Artenliste, Verbreitung, Gefährdung. Hessisches Ministerium für Umwelt, Energie, Landwirtschaft und Verbraucherschutz, 240 p.

Treiber R., 2010. Wildbienen und aculeate Wespen der Rheinaue und rheinnaher Gebiete der elsässischen Oberrheinebene (Dep. Bas-Rhin, Dep. Haut-Rhin; Hymenoptera: Apidae, Chrysididae, Vespidae, Sphecidae, Pompilidae, Scoliidae, Masaridae). Mitteilungen des badischen Landesvereins für Naturkunde und Naturschutz, N.F. 21 (1), 113-147.

Tsuneki K., 1960. Biology of the Japanese Crabroninae (Hymenoptera, Sphecidae). Memoirs of the Faculty of Liberal Arts, Fukui University, (2) 10, 1-53.

Tumšs V., Maršakovs V., 1970. Materiāli Latvijas racējlapseṇu (Hymenoptera, Sphecidae) faunai II. Zoologijas muzeja raksti, 6, 19-33.

Vago J.-L., 2005. Deux Sphecidae Crabroninae peu communs observés dans l'Aisne: Ectemnius nigritarsus (Herrich-Schaeffer) et Lindenius subaeneus Lepeletier \& Brullé (Hymenoptera). Bulletin de la Société entomologique du Nord de la France, 316, 19.

Vago J.-L., 2007. Les Hyménoptères vespiformes (Hymenoptera Tiphiidae, Mutillidae, Scoliidae, Sapygidae, Vespidae, Eumenidae, Pompilidae et Sphecidae) du Nord de la France, un patrimoine à préserver. Le Héron, 40 (4), 173-180.

Vieujant R., 1951. Hyménoptères intéressants pour la faune belge. Bulletin et Annales de la Société Entomologique de Belgique, 87, 146.

Wiesbauer H., Rosa P. \& Zettel H., 2020. Die Goldwespen Mitteleuropas. Biologie, Lebensräume, Artenporträts. E. Ulmer, $254 \mathrm{p}$.

Wiśniowski B., 2004. Annotated checklist of the Polish digger wasps (Hymenoptera: Sphecidae). Polskie Pismo Entomologiczne, 73, 33-63.

Woidak H., 1996. Hymenoptera Aculeata Westfalica. Familia : Sphecidae (Grabwespen). Abhandlungen aus dem Westfälischen Museum für Naturkunde 58 (3), 1-135.

Yıldırım E., Ljubomirov T. \& Lelej A.S., 2014. Overview of the distribution and biogeography of Crabronidae in Turkey (Hymenoptera: Aculeata). Journal of Insect Biodiversity, 2 (3), 1-27. 
Yu D.S., van Achterberg K. \& Horstmann K., 2005. World Ichneumonoidea 2004 - Taxonomy, Biology, Morphology and Distribution. DVD/CD. Taxapad. Vancouver, Canada.

Zettel H., Ockermüller E. \& Wiesbauer H., 2014. Weitere interessante Funde von Grabwespen (Hymenoptera: Sphecidae, Crabronidae) aus Wien und Niederösterreich. Beiträge zur Entomofaunistik, 14, 159-175.

\section{(56 REF)}

